\title{
Photodynamic Therapy with Indocyanine Green Injection and Near-Infrared Light Irradiation Has Phototoxic Effects and Delays Paralysis in Spinal Metastasis
}

\author{
Toru Funayama, M.D., ${ }^{1}$ Masataka Sakane, M.D., Ph.D., ${ }^{1}$ Tetsuya Abe, M.D., Ph.D., ${ }^{2}$ \\ and Naoyuki Ochiai, M.D., Ph.D.1
}

\begin{abstract}
Objective: The purpose of this study was to investigate the phototoxic effects of photodynamic therapy (PDT) with indocyanine green (ICG) and near-infrared light irradiation on rat mammary adenocarcinoma cells, and its therapeutic efficacy in a rat model of spinal metastasis. Background data: Although PDT has been successfully used as a non-radiation treatment for many malignancies, it has not yet been clinically applied for treating spinal metastasis. Methods: For the phototoxicity study, CRL-1666 cells were treated with PDT and cell viability was measured by WST-1 assay. For the efficacy study, 26 female Fischer 344 rats with spinal metastasis in the L6 vertebra were divided into three treatment groups: PDT with local injection of ICG (9 rats), PDT with systemic injection of ICG (10 rats), and no treatment or control (7 rats). Both the PDT groups received near-infrared light irradiation with a total energy of $10 \mathrm{~J}(1 \mathrm{~W}$ for $10 \mathrm{sec})$. The light was delivered directly through a single silica probe which was set on the left side of the L6 vertebral body. Hindlimb motor function was monitored according to the Basso-Beattie-Bresnahan (BBB) scale. Further, the observation periods were calculated to determine the survival time. Results: The PDT exerted immediate and persistent phototoxic effects. Furthermore, the PDT with local injection of ICG as well as systemic injection of ICG delayed the deterioration of paralysis and prolonged the observation period. Conclusions: PDT with ICG injection and near-infrared light irradiation could be an effective local adjuvant treatment for spinal metastasis.
\end{abstract}

\section{Introduction}

$\mathbf{M}$ ETASTASIS TO BONE is a major cause of pain and disability. The spinal column is a common site of bone metastasis in $\sim 40 \%$ of the patients with cancer. ${ }^{1}$ Among these, breast cancer is the most frequent origin. ${ }^{2,3}$ Metastatic epidural spinal cord compression (MESCC) is a debilitating complication of spinal metastasis occurring in $\sim 5-20 \%$ of the patients with cancer. ${ }^{1,4-6}$ Because the survival time of patients with metastatic cancer is increasing, the incidence of symptomatic spinal metastatic disease is expected to increase as well. Local control of spinal metastasis is important because MESCC is significantly associated with the quality of life (QOL) of these patients. ${ }^{7}$ Although advanced surgery has been performed in patients with spinal metastasis 8,9 and has improved the patients' QOL, ${ }^{10,11}$ local recurrence is relatively common $^{4,12}$ and the therapeutic options after recurrence are often limited to such as radiation therapy. ${ }^{4}$ Therefore, there is an urgent need for novel and more effective treatment modalities.
Photodynamic therapy (PDT) has been successfully applied as a non-radiation treatment for many malignancies. ${ }^{13,14}$ Although PDT for spinal metastasis has been studied experimentally, ${ }^{15-17}$ it has not yet been clinically applied.

Indocyanine green (ICG) is widely used in the clinical setting for liver function tests, ${ }^{18}$ cardiac output measurement, ${ }^{19}$ and ophthalmic angiography. ${ }^{20}$ It is a tricarbocyanine dye and exhibits an absorption maximum at $\sim 810$-nm wavelength of light. ${ }^{21}$ The phototoxic effect of ICG-enhanced therapy has been studied in various fields. ${ }^{22-28}$ However, the strong binding of plasma proteins with ICG and its subsequent rapid clearance by the liver ${ }^{21}$ results in no tumor selectivity after systemic administration.

The aim of the present study was to investigate the phototoxic effects of PDT with ICG administration and nearinfrared light irradiation on rat mammary adenocarcinoma cells, and its therapeutic efficacy in a rat model of spinal metastasis. We hypothesized that if the PDT had significant phototoxic effects in vitro, it would be an effective local

\footnotetext{
${ }^{1}$ Department of Orthopaedic Surgery, Graduate School of Comprehensive Human Sciences, University of Tsukuba, Tsukuba, Ibaraki, Japan.

${ }^{2}$ Department of Orthopaedic Surgery, Tsukuba Central Hospital, Ushiku, Ibaraki, Japan.
} 
treatment for spinal metastasis and the therapeutic efficacy would depend upon the manner of ICG administration.

\section{Methods}

\section{Tumor cell line}

Rat mammary adenocarcinoma (CRL-1666) was selected and purchased from the American Type Culture Collection (Manassas, VA). The cells were cultured in Dulbecco's modified Eagle's medium (DMEM) containing 10\% fetal bovine serum, $80.5 \mathrm{pg} / \mathrm{mL}$ streptomycin, $80.5 \mathrm{U} / \mathrm{mL}$ penicillin, and $1 \%$ L-glutamine, and maintained in a humidified atmosphere of $5 \% \mathrm{CO}_{2}$ at $37^{\circ} \mathrm{C}$. For the experiments, cells in the exponential growth phase were used.

\section{Animal care}

Twenty-six Fischer 344 rats weighing 130-150g were purchased from Oriental Bio Service Kanto Co., Ltd. (Tsukuba, Japan) for the experiments. All animals were cared for in accordance with the guidelines of the Laboratory Animal Resource Center of University of Tsukuba. The rats were anesthetized by intraperitoneal injection of sodium pentobarbital (Somnopentil, $32.5 \mathrm{mg} / \mathrm{kg}$ body weight) at the time of the experiments.

\section{Instruments and materials}

The light irradiation was administered via a near-infrared medical diode laser (UDL-15, Olympus, Tokyo, Japan; $\lambda=810 \pm 20 \mathrm{~nm}$, maximum power output $=15 \mathrm{~W})$. The output beam of the laser was directly through a single silica probe (DM-6065, Olympus, Tokyo, Japan; core diameter $=600 \mu \mathrm{m}$ ). ICG (Diagnogreen, Daiichi-Sankyo, Tokyo, Japan) was used as the photosensitizer in the present study.

\section{Phototoxicity study}

The adenocarcinoma cells were seeded into 16 wells $\left(2.0 \times 10^{4}\right.$ cells / well) of 96-well plates (IWAKI, Tokyo, Japan) with $100 \mu \mathrm{L}$ medium in each well and incubated overnight. The plates were divided into the following groups: control or untreated group, ICG administration alone (ICG group), laser irradiation alone (laser group), and ICG administration plus laser irradiation (PDT group). The control and laser groups were incubated in medium, and the ICG and PDT groups were incubated in $64.5 \mu \mathrm{M}$ ICG (dissolved in medium) for $24 \mathrm{~h}$. On the next day, the laser and PDT groups were treated with laser irradiation (power output $=10 \mathrm{~W}$ for $30 \mathrm{sec}$; total energy density $=18.8 \mathrm{~J} / \mathrm{cm}^{-2}$ ). The lid of the each 96-well plate was removed during irradiation and the laser probe was set above the plate to irradiate all of the 16 wells at once. The light was irradiated for $30 \mathrm{sec}$ in continuous mode and automatically turned off after the irradiation. Each well was washed with phosphate-buffered saline before the irradiation, replaced with fresh medium after the irradiation, and then incubated for 0,24 , 48 , or $72 \mathrm{~h}$. Furthermore, the plates of the laser and PDT groups were incubated for $96 \mathrm{~h}$. In total, 18 plates were used, and the experiment was performed in parallel wells using separate plates for each experimental condition.

\section{WST-1 assay}

Cell viability after each treatment was determined by WST-1 assay (Roche Diagnostics Japan, Tokyo, Japan). After the medium in the wells was renewed, $10 \mu \mathrm{L}$ of WST- 1 was added to each well and the cells were incubated for $1 \mathrm{~h}$ to terminate the reaction. The absorbance of the formazan product was measured as the optical density at $450 \mathrm{~nm}$ with a microplate reader (MTP-300, Corona Electric, Hitachinaka, Japan).

\section{PDT efficacy study}

A rat model of spinal metastasis ${ }^{29}$ was selected to evaluate the therapeutic efficacy of the PDT. After exposing the L6 vertebral body by a direct abdominal approach, an 18-gauge needle was used to create a hole in the L6 vertebral body $\sim 1 \mathrm{~mm}$ to the left of the midline. Once the desired depth $(1.5 \mathrm{~mm})$ was achieved, the needle was rotated to create a 1mm-wide hole to implant a tumor section $(\sim 1 \times 1 \times 1 \mathrm{~mm})$ with microsurgical instruments. The tumor section was obtained from a rat with a subcutaneous tumor of CRL-1666 which was prepared prior to the experiment. ${ }^{29}$ After the implantation, the hole was sealed with a hydroxyapatite bead $($ diameter $=\sim 1.7 \mathrm{~mm}){ }^{30}$

On day 7 after the implantation, the animals were anesthetized and their abdomens were exposed again by using the same approach as for the implantation. The hydroxyapatite bead overlying the previous implantation site was removed carefully. The rats were then divided into the following three treatment groups: PDT with local injection of ICG (PDT-L group, 9 rats), PDT with systemic injection of ICG (PDT-V group, 10 rats), and control or no treatment (control group, 7 rats). In the PDT-L group, the rats received a $5 \mu \mathrm{L}$ injection of $25 \mathrm{mg} / \mathrm{mL}$ ICG in the hole of the L6 vertebral body with a $10 \mu \mathrm{L}$ Gastight Hamilton Syringe and the light irradiation with a total energy of $10 \mathrm{~J}$ ( $1 \mathrm{~W}$ for $10 \mathrm{sec})$ immediately. The probe was set on the left side of the L6 vertebral body and touched the hole during the irradiation. In the PDT-V group, the rats received a $0.2-\mathrm{mL}$ intravenous injection of $25 \mathrm{mg} / \mathrm{mL}$ ICG via the tail vein, and the light irradiation with the same energy as the PDT-L group $3 \mathrm{~min}$ after the ICG injection. In the control group, the rats received mock irradiation. The animals were allowed to recover after the treatment.

\section{Assessment of motor function}

Hindlimb motor function was monitored daily according to the Basso-Beattie-Bresnahan (BBB) scale. ${ }^{31}$ The BBB scale ranges from 0 to 21 points for the characteristics of gait. The rats were placed in a molded plastic circular enclosure $(57 \times 38 \times 30 \mathrm{~cm})$ and allowed to become accustomed to their surroundings. For determining the BBB scores, the animals were observed individually for $4 \mathrm{~min}$ by 2 observers blinded to the treatment allocation.

\section{Assessment of survival and histology}

According to the institutional review board criteria, the animals were euthanized by intraperitoneal injection of $130.0 \mathrm{mg} / \mathrm{kg}$ body weight of sodium pentobarbital (Somnopentil) with a 25-gauge needle if their BBB scores fell $<3$; otherwise they were allowed to die naturally. Therefore, the end point of the experiments was defined as the day of euthanasia or death and the observation period was defined as the duration from the day of tumor implantation to the day 
FIG. 1. Results of the phototoxicity study. The bar graph shows the mean absorbance (optical density) measured by the WST-1 assay (mean $\pm \mathrm{SD}, \quad{ }^{*} p<0.01$, $n=16$, n.s. $=$ not significant).

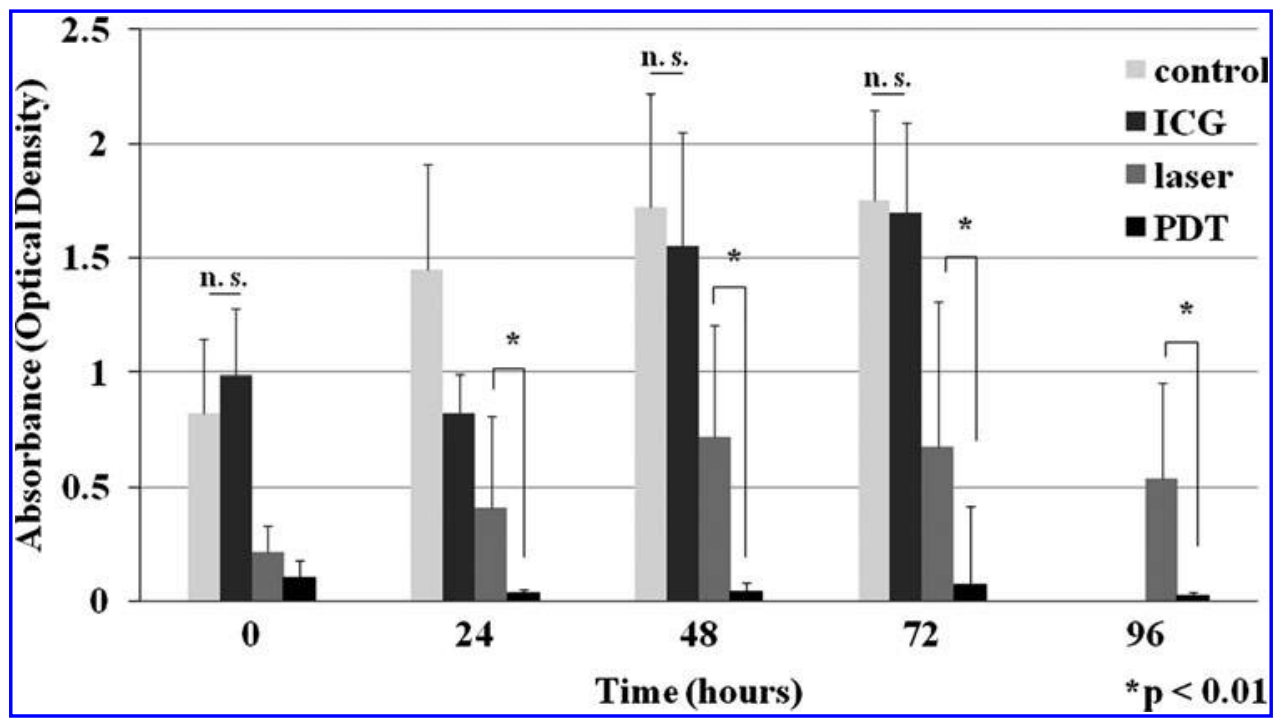

of the end point. At the end of the experiments, an autopsy was conducted to examine the spine. Dissected spines were fixed in $10 \%$ formalin solution, decalcified, and processed for hematoxylin \& eosin (H\&E) staining. Axial cross sections of the spine were obtained, and vertebral invasion and epidural spinal cord compression were histologically inspected.

\section{Statistical analysis}

The mean absorbance in the WST-1 assay (each time point of each group, $n=16$ ) was analyzed by using ANOVA for comparison of multiple groups. The mean BBB scores were compared by using the Wilcoxon signed rank test. The observation period was determined by using Kaplan-Meier analysis and the log-rank test. Values of $p<0.05$ were considered statistically significant.

\section{Results}

\section{Phototoxicity}

In the WST-1 assay (Fig. 1), the PDT group had an approximately eightfold decrease in optical density compared with the control and ICG groups at $0 \mathrm{~h}$ (PDT group, $0.10 \pm 0.08$; control group, $0.82 \pm 0.32$ ). The optical density of the PDT group as well as the laser group was significantly lower than that of the control group at all time points until $72 \mathrm{~h}$. Further, the optical density of the PDT group was significantly lower than that of the laser group after $24 \mathrm{~h}$. From 24 to $96 \mathrm{~h}$, the optical density of the PDT group showed no statistical difference. The cells in the control and ICG groups showed a linear growth pattern and reached confluence after $72 \mathrm{~h}$; there was no significant difference between these groups at 0,48 , and $72 \mathrm{~h}$.

\section{Efficacy of PDT}

The functional outcome of the animals was tracked by using the BBB scale (Fig. 2). Before and after the tumor implantation until day 6, all the animals displayed a BBB score of 21 (normal hindlimb function). One rat in each group displayed a BBB score of 19 (inability to raise the tail) on day 7. Therefore, the mean pretreatment BBB scores were as follows: PDT-L group, 20.8; PDT-V group, 20.8; control group, 20.7. The mean BBB scores of the PDT groups were

FIG. 2. Functional outcomes after PDT. The line graph shows the average $\mathrm{BBB}$ scores of the three groups of rats throughout the investigation.

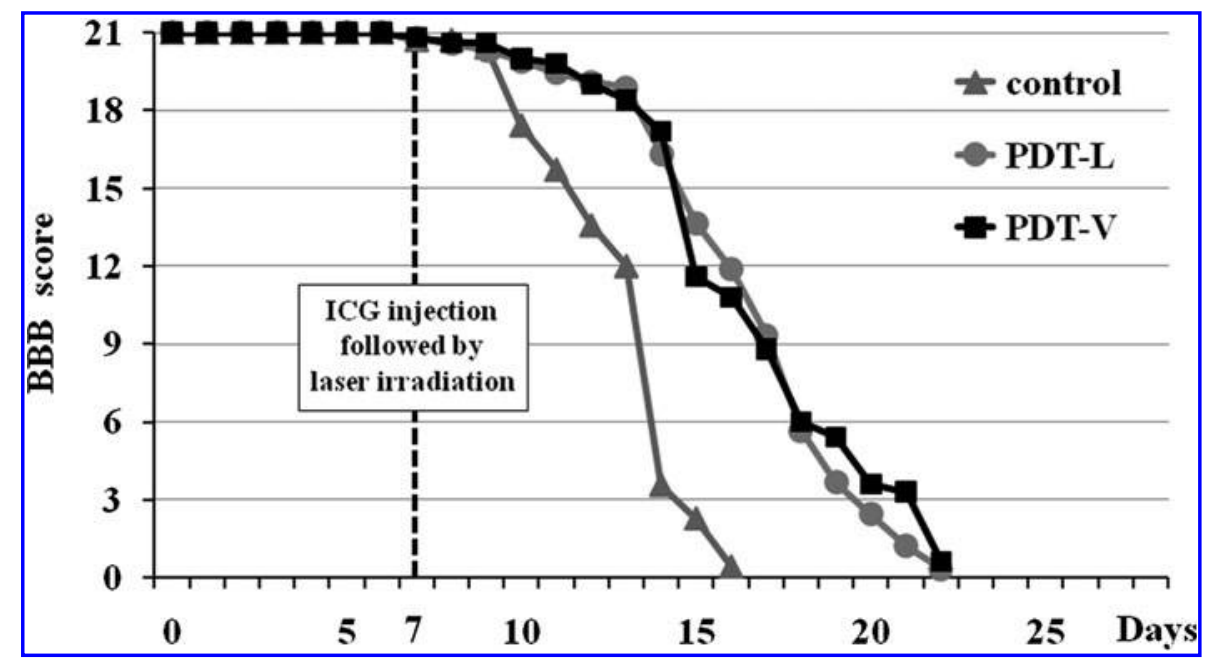




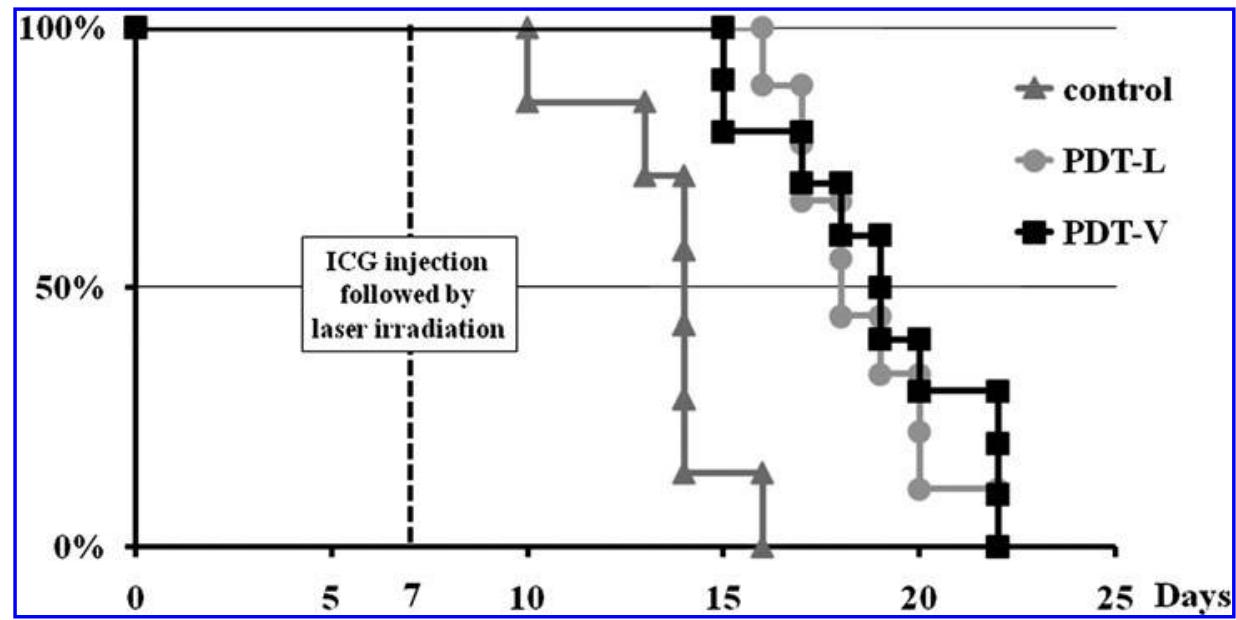

FIG. 3. Mortality after PDT. The line graph shows the Kaplan-Meier survival curves of the three groups of rats during the investigation.

$>18.0$ until day 13 after the implantation, but the mean BBB score of the control group decreased rapidly after day 10 from the implantation. Further, the mean BBB scores of the PDT-L and PDT-V groups were significantly higher than that of the control group from days 13 and 11 after the implantation, respectively. No statistically significant difference was found between the PDT-L and the PDT-V groups.

The mean observation periods were 18.6, 18.9, and 13.6 days after the implantation in the PDT-L, PDT-V, and control groups, respectively. The Kaplan-Meier analysis showed that the PDT-L and PDT-V groups had significantly longer (PDT-L, $p=0.002$; PDT-V, $p=0.001$ ) observation periods than did the control group (Fig. 3). By day 16 after the implantation, all the surviving animals of the control group reached the end point of the experiments, whereas the surviving animals from both the PDT groups reached the end point of the experiment by day 22. One rat in the control group did not develop paralysis, but it died of ascites carcinoma because of the extraosseous tumor burden. Except for this animal, all the other animals developed complete paralysis. There was no significant difference in the observation periods between the PDT-L and the PDT-V groups.

The tumor often grew ventrally, leading to ascites carcinoma. Microscopic examinations of the axial cross sections of the L6 vertebra revealed that the CRL-1666 adenocarcinoma cells filled the bone marrow spaces, destroyed trabecular bone structures of the spinal canal, and compressed the spinal cord in the control group (Fig. 4). On the other hand, the cancer cells were not seen in the vertebral body in either of the PDT groups. The tumor cells beyond the limits of the laser irradiation developed the lesion, invaded the spinal canal, and finally compressed the spinal cord (Fig. 4).

\section{Discussion}

Considering the results of this study, the PDT with ICG administration and near-infrared light irradiation had a persistent phototoxic effect on the rat mammary adenocarcinoma cells in vitro. Furthermore, the PDT with local injection of ICG as well as systemic injection of ICG and near-infrared light irradiation delayed the deterioration of paralysis and prolonged the observation period in the rat model of spinal metastasis. The PDT effectively killed the
CRL-1666 cancer growing in the L6 vertebral body of the rats in the area of irradiation. This study was the first report on therapeutic effects of PDT for motor function in the rat model of spinal metastasis.

The in vitro effects of near-infrared light irradiation on cell survival have been investigated on different cell lines. ${ }^{32,33}$ High energy and power densities of the light can produce damaging amounts of reactive oxygen species (ROS) that decrease cell viability. In PDT, when the photosensitizer is exposed to the appropriate wavelength of light, it is activated from a ground state to an excited state. As it returns to the ground state, it releases energy, which is transferred to oxygen molecules, generating ROS, such as singlet oxygen and free radicals; these ROS mediate cellular toxicity. ${ }^{14}$ The in vitro effects of PDT with ICG and near-infrared light irradiation on cell survival have also been investigated. ${ }^{22,26}$ Similarly, when ICG is exposed to near-infrared light, singlet oxygen is generated, ${ }^{34}$ ICG is decomposed by singlet oxygen itself, and the decomposition products further decrease cell viability. ${ }^{35}$

We also investigated the efficacy of the PDT with ICG injection and near-infrared light irradiation in rats with spinal metastasis. The PDT improved the hindlimb function and prolonged the observation period. As per the previous studies using the same animal model, ${ }^{36-38}$ we treated the rats 7 days after the tumor implantation. We consider that the timing of treatment in this animal model simulates the beginning of paralysis because of MESCC. Several studies of local treatment for spinal metastasis have been reported by using this well-established model. ${ }^{30,36-41}$ The power of irradiation and the amount of ICG injection were determined according to the results of a preliminary safety test in normal rats. There was no iatrogenic paralysis following the irradiation with a total energy of $10 \mathrm{~J}$ ( $1 \mathrm{~W}$ for $10 \mathrm{sec})$ or any complication after the ICG injection. The histological inspection of the preliminary safety test in four normal rats revealed that the depth of the histological change was within $\sim 2 \mathrm{~mm}$ from the irradiation site. Therefore, the neural tissue in the canal spaces was intact in all of the rats (histology was not shown). Considering that the hindlimb function was maintained for $\sim 7$ days after the PDT and paralysis was delayed in the treated rats, the PDT may be an effective local treatment for spinal metastasis. In the untreated group, intra-osseous tumor-bearing rats 
FIG. 4. Histological examination of the spine (H\&E staining). (a) and (b) Axial cross-section of a rat in the control group. The CRL1666 adenocarcinoma cells implanted in the hole $(\mathrm{H})$ of the L6 vertebral body (B) filled the bone marrow spaces and developed osteolytic lesions. The tumor destroyed the structure of the posterior wall $(\mathrm{P})$, invaded the spinal canal $(\mathrm{M})$, and compressed the spinal cord (C) severely. (c) and (d) Axial cross-section of a rat in the PDT-L group. PDT delayed the epidural spinal cord compression for an average of 5 days. The CRL-1666 adenocarcinoma cells were not seen in the vertebral body (B). However, the tumor cells beyond the limits of the laser irradiation developed the lesion, invaded the spinal canal $(\mathrm{M})$, and finally compressed the spinal cord (C). (e) and (f) Axial cross-section of a rat in the PDT-V group. Histological findings were almost the same as for those in the PDT-L group.

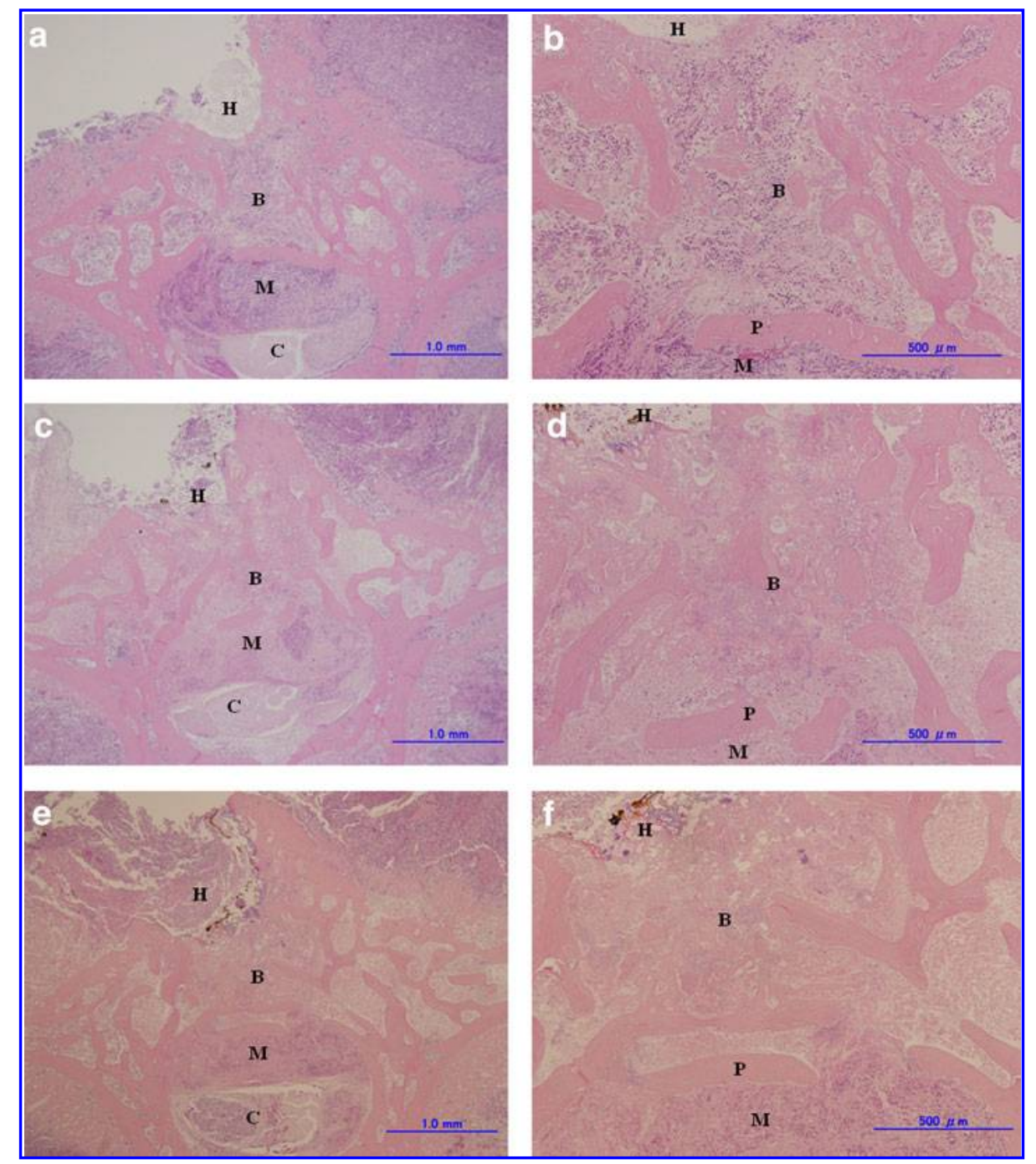

developed hindlimb paralysis at 7-13 days and reached the end point of the experiment $<16$ days after the tumor implantation.

The limitation of the in vivo study was that the therapeutic efficacy of the near-infrared light irradiation alone was not studied. Although the PDT with ICG and nearinfrared light irradiation had phototoxic effects, the conditions of ICG administration were different between in vitro and in vivo studies. Therefore, the results of the phototoxicity study could not be replaced as the therapeutic efficacy study. Further studies are required to clarify the therapeutic efficacy of near-infrared light irradiation alone as a control.

In the present study, both local injection of ICG and intravenous injection of ICG had therapeutic effects on the hindlimb function and the observation period. The efficacy of PDT did not depend upon the manner of ICG administration. When injected intravenously, ICG spreads to the whole body first. Subsequently, hepatic uptake begins rapidly and the fraction dose of ICG decreases to $\sim 0.1$ within 5 min in rats. ${ }^{42}$ Furthermore, although locally injected ICG was administered correctly by using the Gastight Hamilton Syringe, there was no way to detect the spread area of ICG in each rat. Although further studies are needed to clarify the dose of ICG administration and the timing of near-infrared light irradiation, PDT with local injection of ICG as well as systemic injection of ICG and near-infrared light irradiation could be an effective local treatment for spinal metastasis. In clinical settings, it is essential that a photosensitizer should have locally and selectively accumulated in the lesion for safe PDT. Therefore, we believe that a carrier that can deliver ICG to the metastatic lesions selectively is needed for further investigation.

During resection of a malignant tumor, it is necessary to remove the normal margin as well. However, marginal resection is often difficult in spinal metastasis because important tissues such as the aorta, vena cava, and dura mater including the spinal cord are located adjacent to the vertebral body. We consider that the PDT method could be applied for the marginal lesions, to reduce the local recurrence. Local adjuvant PDT combined with open surgery may reduce the morbidity associated with spinal metastasis. Furthermore, recently there has been considerable focus on the potential use of ICG in intra-operative real-time imaging. ${ }^{43-49}$ The combined use of ICG and near-infrared light could be a novel therapeutic system, including intra-operative imaging modalities and PDT. 


\section{Conclusions}

The PDT with ICG and near-infrared light irradiation had an immediate and persistent phototoxic effect on the rat mammary adenocarcinoma cells. Furthermore, the PDT with local injection of ICG as well as systemic injection of ICG and near-infrared light irradiation delayed the deterioration of paralysis and prolonged the observation period in the rat model of spinal metastasis. The results indicate that such PDT could be an effective local treatment for spinal metastasis and therefore improve the QOL of patients with spinal metastasis.

\section{Acknowledgments}

This study was partially supported by a grant from the Japan Science and Technology Agency, Innovation Satellite Ibaraki, Practical Application Research.

\section{Author Disclosure Statement}

No conflicting financial interests exist.

\section{References}

1. Klimo, P., Jr., and Schmidt, M.H. (2004). Surgical management of spinal metastases. Oncologist 9, 188-196.

2. Bohm, P., and Huber, J. (2002). The surgical treatment of bony metastases of the spine and limbs. J. Bone Joint Surg. Br. 84, 521-529.

3. Schaberg, J. and Gainor, B.J. (1985). A profile of metastatic carcinoma of the spine. Spine. 10, 19-20.

4. Cole, J.S., and Patchell, R.A. (2008). Metastatic epidural spinal cord compression. Lancet Neurol. 7, 459-466.

5. Quraishi, N.A., Gokaslan, Z.L., and Boriani, S. (2010). The surgical management of metastatic epidural compression of the spinal cord. J. Bone Joint. Surg. Br. 92, 1054-1060.

6. Oka, H., Kondoh, T., Seichi, A., Hozumi, T., and Nakamura, K. (2006). Incidence and prognostic factors of Japanese breast cancer patients with bone metastasis. J. Orthop. Sci. $11,13-19$.

7. Abrahm, J.L., Banffy, M.B., and Harris, M.B. (2008). Spinal cord compression in patients with advanced metastatic cancer: "all I care about is walking and living my life". JAMA 299, 937-946.

8. Tomita, K., Kawahara, N., Murakami, H., and Demura, S. (2006). Total en bloc spondylectomy for spinal tumors: improvement of the technique and its associated basic background. J. Orthop. Sci. 11, 3-12.

9. Tomita, K., Toribatake, Y., Kawahara, N., Ohnari, H., and Kose, H. (1994). Total en bloc spondylectomy and circumspinal decompression for solitary spinal metastasis. Paraplegia 32, 36-46.

10. Patchell, R.A., Tibbs, P.A., Regine, W.F., Payne, R., Saris, S., Kryscio, R.J., et al. (2005). Direct decompressive surgical resection in the treatment of spinal cord compression caused by metastatic cancer: a randomised trial. Lancet 366, 643-648.

11. Ibrahim, A., Crockard, A., Antonietti, P., Boriani, S., Bunger, C., Gasbarrini, A., et al. (2008). Does spinal surgery improve the quality of life for those with extradural (spinal) osseous metastases? An international multicenter prospective observational study of 223 patients. Invited submission from the Joint Section Meeting on Disorders of the Spine and Peripheral Nerves, March 2007. J. Neurosurg. Spine. 8, 271278.
12. Sakaura, H., Hosono, N., Mukai, Y., Ishii, T., Yonenobu, K., and Yoshikawa, H. (2004). Outcome of total en bloc spondylectomy for solitary metastasis of the thoracolumbar spine. J. Spinal Disord. Tech. 17, 297-300.

13. Brown, S.B., Brown, E.A., and Walker, I. (2004). The present and future role of photodynamic therapy in cancer treatment. Lancet Oncol. 5, 497-508.

14. Dolmans, D.E., Fukumura, D., and Jain, R.K. (2003). Photodynamic therapy for cancer. Nat. Rev. Cancer 3, 380-387.

15. Akens, M.K., Hardisty, M.R., Wilson, B.C., Schwock, J., Whyne, C.M., Burch, S., et al. (2010). Defining the therapeutic window of vertebral photodynamic therapy in a murine pre-clinical model of breast cancer metastasis using the photosensitizer BPD-MA (Verteporfin). Breast Cancer Res. Treat. 119, 325-333.

16. Akens, M.K., Yee, A.J., Wilson, B.C., Burch, S., Johnson, C.L., Lilge, L., et al. (2007). Photodynamic therapy of vertebral metastases: evaluating tumor-to-neural tissue uptake of BPD-MA and ALA-PpIX in a murine model of metastatic human breast carcinoma. Photochem Photobiol. 83, 10341039.

17. Burch, S., Bisland, S.K., Bogaards, A., Yee, A.J., Whyne, C.M., Finkelstein, J.A., et al. (2005). Photodynamic therapy for the treatment of vertebral metastases in a rat model of human breast carcinoma. J. Orthop. Res. 23, 995-1003.

18. Caesar, J., Shaldon, S., Chiandussi, L., Guevara, L., and Sherlock, S. (1961). The use of indocyanine green in the measurement of hepatic blood flow and as a test of hepatic function. Clin. Sci. 21, 43-57.

19. Maarek, J.M., Holschneider, D.P., and Rubinstein, E.H. (2007). Fluorescence dilution technique for measurement of cardiac output and circulating blood volume in healthy human subjects. Anesthesiology 106, 491-498.

20. Slakter, J.S., Yannuzzi, L.A., Guyer, D.R., Sorenson, J.A., and Orlock, D.A. (1995). Indocyanine-green angiography. Curr. Opin. Ophthalmol. 6, 25-32.

21. Cherrick, G.R., Stein, S.W., Leevy, C.M., and Davidson, C.S. (1960). Indocyanine green: observations on its physical properties, plasma decay, and hepatic extraction. $\underline{\text { J. Clin. }}$ Invest. 39, 592-600.

22. Bozkulak, O., Yamaci, R.F., Tabakoglu, O., and Gulsoy, M. (2009). Photo-toxic effects of 809-nm diode laser and indocyanine green on MDA-MB231 breast cancer cells. Photodiagnosis Photodyn. Ther. 6, 117-121.

23. Kim, B.J., Lee, H.G., Woo, S.M., Youn, J.I., and Suh, D.H. (2009). Pilot study on photodynamic therapy for acne using indocyanine green and diode laser. I. Dermatol. 36, 17-21.

24. Noritomi, T., Yamashita, Y., Kodama, T., Mikami, K., Hashimoto, T., Konno, T., et al. (2005). Application of dyeenhanced laser ablation for liver resection. Production of protein sealant on the cut surface of the liver by enhanced thermal energy of low power diode laser. Eur. Surg. Res. 37, 153-158.

25. Sato, M., Ishihara, M., Arai, T., Asazuma, T., Kikuchi, T., Hayashi, T., et al. (2001). Use of a new ICG-dye-enhanced diode laser for percutaneous laser disc decompression. Lasers Surg. Med. 29, 282-287.

26. Skrivanova, K., Skorpikova, J., Svihalek, J., Mornstein, V., and Janisch, R. (2006). Photochemical properties of a potential photosensitiser indocyanine green in vitro. J. Photochem. Photobiol. B. 85, 150-154.

27. Abels, C., Fickweiler, S., Weiderer, P., Baumler, W., Hofstadter, F., Landthaler, M., et al. (2000). Indocyanine green (ICG) and laser irradiation induce photooxidation. Arch. Dermatol. Res. 292, 404-411. 
28. Baumler, W., Abels, C., Karrer, S., Weiss, T., Messmann, H., Landthaler, M., et al. (1999). Photo-oxidative killing of human colonic cancer cells using indocyanine green and infrared light. Br. J. Cancer 80, 360-363.

29. Mantha, A., Legnani, F.G., Bagley, C.A., Gallia, G.L., Garonzik, I., Pradilla, G., et al. (2005). A novel rat model for the study of intraosseous metastatic spine cancer. J. Neurosurg. Spine 2, 303-307.

30. Abe, T., Sakane, M., Ikoma, T., Kobayashi, M., Nakamura, S., and Ochiai, N. (2008). Intraosseous delivery of paclitaxelloaded hydroxyapatitealginate composite beads delaying paralysis caused by metastatic spine cancer in rats. I. Neurosurg. Spine 9, 502-510.

31. Basso, D.M., Beattie, M.S., and Bresnahan, J.C. (1995). A sensitive and reliable locomotor rating scale for open field testing in rats. J. Neurotrauma. 12, 1-21.

32. Karu, T. (1999). Primary and secondary mechanisms of action of visible to near-IR radiation on cells. I. Photochem. Photobiol. B. 49, 1-17.

33. Lavi, R., Shainberg, A., Shneyvays, V., Hochauser, E., Isaac, A., Zinman, T., et al. (2010). Detailed analysis of reactive oxygen species induced by visible light in various cell types. Lasers Surg. Med. 42, 473-480.

34. Hirano, T., Kohno, E., Gohto, Y., and Obana, A. (2006). Singlet oxygen generation due to ICG irradiation. Photomed. Photobiol. 28, 15-16.

35. Engel, E., Schraml, R., Maisch, T., Kobuch, K., Konig, B., Szeimies, R.M., et al. (2008). Light-induced decomposition of indocyanine green. Invest. Ophthalmol. Vis. Sci. 49, 1777-1783.

36. Bagley, C.A., Bookland, M.J., Pindrik, J.A., Ozmen, T., Gokaslan, Z.L., Wolinsky, J.P., et al. (2007). Fractionated, single-port radiotherapy delays paresis in a metastatic spinal tumor model in rats. J. Neurosurg. Spine. 7, 323-327.

37. Gok, B., McGirt, M., Sciubba, D.M., Ayhan, S., Bydon, A., Witham, T.F., et al. (2008). Surgical resection plus adjuvant radiotherapy is superior to surgery or radiotherapy alone in the prevention of neurological decline in a rat metastatic spinal tumor model. Neurosurgery 63, 346-351; discussion 351.

38. Gok, B., McGirt, M.J., Sciubba, D.M., Garces-Ambrossi, G., Nelson, C., Noggle, J., et al. (2009). Adjuvant treatment with locally delivered OncoGel delays the onset of paresis after surgical resection of experimental spinal column metastasis. Neurosurgery 65, 193-199; discussion 199-200.

39. Abe, T., Sakane, M., Ikoma, T., Kobayashi, M., and Ochiai, N. (2009). Therapeutic effects of paclitaxel-loaded hydroxyapatite-alginate beads: in vitro and in vivo studies. Key Eng. Mater. 396-398, 551-554.

40. Abe, T., Sakane, M., Ikoma, T., Tonegawa, T., Yoshioka, T., Tanaka, J., et al. (2007). Novel local treatment with pacli- taxel-loaded hydroxyapatite-alginate gels for spinal bone metastases. Key Eng. Mater. 330-332, 1343-1346.

41. Bagley, C.A., Bookland, M.J., Pindrik, J.A., Ozmen, T., Gokaslan, Z.L., and Witham, T.F. (2007). Local delivery of oncogel delays paresis in rat metastatic spinal tumor model. I. Neurosurg. Spine. 7, 194-198.

42. Scharschmidt, B.F., Waggoner, J.G., and Berk, P.D. (1975). Hepatic organic anion uptake in the rat. J. Clin. Invest. 56, 1280-1292.

43. Colby, G.P., Coon, A.L., Sciubba, D.M., Bydon, A., Gailloud, P., and Tamargo, R.J. (2009). Intraoperative indocyanine green angiography for obliteration of a spinal dural arteriovenous fistula. J. Neurosurg. Spine 11, 705-709.

44. Handa, T., Katare, R.G., Nishimori, H., Wariishi, S., Fukutomi, T., Yamamoto, M., et al. (2010). New device for intraoperative graft assessment: HyperEye charge-coupled device camera system. Gen Thorac Cardiovasc. Surg. 58, 68-77.

45. Marshall, M.V., Rasmussen, J.C., Tan, I.-C., Aldrich, M.B., Adams, K.E., Wang, X., et al. (2010). Near-infrared fluorescence imaging in humans with indocyanine green: a review and update. Open Surg. Oncol. J. 2, 12-25.

46. Oh, J.K., Shin, H.C., Kim, T.Y., Choi, G.H., Ji, G.Y., Yi, S., et al. (2011). Intraoperative indocyanine green videoangiography: spinal dural arteriovenous fistula. Spine [Epub ahead of print].

47. Schaafsma, B.E., Mieog, J.S., Hutteman, M., van der Vorst, J.R., Kuppen, P.J., Lowik, C.W., et al. (2011). The clinical use of indocyanine green as a near-infrared fluorescent contrast agent for image-guided oncologic surgery. J. Surg Oncol. 104, 323-332.

48. Schubert, G.A., Barth, M., and Thome, C. (2010). The use of indocyanine green videography for intraoperative localization of intradural spinal tumors. Spine [Epub ahead of print].

49. Schubert, G.A., Schmieder, K., Seiz-Rosenhagen, M., and Thome, C. (2011). ICG videography facilitates interpretation of vascular supply and anatomical landmarks in intramedullary spinal lesions: two case reports. Spine 36, E811-813.

Address correspondence to: Masataka Sakane 1-1-1 Tennoudai Tsukuba Ibaraki, 305-8575 Japan

E-mail: sakane-m@md.tsukuba.ac.jp 
